03

THE UK PAEDIATRIC LIVER TRANSPLANT PROGRAMME DURING THE COVID-19 PANDEMIC

${ }^{1}$ Tassos Grammatikopoulos, ${ }^{2}$ Rhiannon Taylor, ${ }^{2}$ Julie Whitney, ${ }^{3}$ Jane Hartley, ${ }^{4}$ Magdy Attia ${ }^{5}$ Darius Mirza, ${ }^{6}$ Sanjay Rajwal, ${ }^{7}$ Sarah Watson, ${ }^{2}$ John Isaac, ${ }^{2}$ Derek Manas, ${ }^{8}$ Nigel Heaton, ${ }^{1}$ Anil Dhawan, ${ }^{2}$ John Forsythe, ${ }^{2}$ Douglas Thorburn. ${ }^{1}$ Paediatric Liver, Gl and Nutrition Centre and Mowat Labs, King's College Hospital; ${ }^{2}$ NHS Blood and Transplant; ${ }^{3}$ Birmingham Children's Hospital; ${ }^{4}$ The Liver Transplant Unit, Leeds Teaching Hospitals Trust; ${ }^{5}$ The Liver Unit, Queen Elizabeth Hospital Birmingham; ${ }^{6}$ Children's Liver Unit, Leeds General Infirmary; ${ }^{7}$ Highly Specialised Services, NHS England and NHS Improvement; ${ }^{8}$ Institute of Liver Studies, King's College Hospital

10.1136/flgastro-2021-bspghan.3

Introduction The UK has been severely affected by the COVID-19 pandemic. The impact on the adult population has been disproportionately higher when compared to children with consequent challenges to organ donation and liver transplantation (LT). Across the three UK paediatric liver centres there has only been a very small number of patients who tested positive for COVID-19 and all made a speedy and full recovery. We report here the response during the pandemic across the 3 paediatric LT centres.

Methods A series of nationally agreed policy changes affecting the liver procurement, listing and transplant process were agreed during regular meetings with LT centre directors and NHSE. Actions at a local and national level were agreed to protect and maintain the paediatric LT programmes.

Data were collected from 27/03/20 until 26/11/20 and compared with same time period for the years 2016-19.

Results During the study period, there was a significant reduction in the adult population in the mean number of weekly liver offers, donors and LTs compared to before the pandemic with signs of recovery between the 1 st and 2nd UK lockdown periods (figure 1). More specifically the number of livers offered nationally was reduced from an average 30-40/week to only <10/week during the 1 st wave in the March-April period. The number of children on the LT list during the study period across all 3 centres was 74 in total with 17 (23\%) super-urgent and 57(77\%) electives, which was comparable to previous years.

Overall, 65-80 paediatric LTs are performed annually across the UK's 3 paediatric centres.

From March-November 2020 there were 58(82\%) elective and $13(18 \%)$ super urgent (acute liver failure \& hepatoblastoma) paediatric LTs performed.

Donor Brain Dead (DBD) and Donor Cardiac Dead (DCDC) LTs were 54(76\%) and 3(4\%), respectively. Living related LT (LRLT) programme was sustained comprising 20\% of LTs performed.

The number of paediatric LTs performed during the pandemic was comparable to those performed yearly since 2016 . The number of LT per paediatric centre for King's College Hospital $(\mathrm{KCH})$, Birmingham Children's Hospital $(\mathrm{BCH})$ and Leeds Liver Unit were 40 (56\%), 15(21\%) and 16(23\%), respectively with excellent outcome. A 15-year-old girl from $\mathrm{KCH}$ diagnosed with Wilson disease presented with liver failure and became COVID-19 positive whilst listed. She underwent LT soon after becoming COVID-19 negative. No perioperative mortality was reported with excellent outcome so far in all.

Conclusion The current COVID-19 pandemic had a significant impact on the UK adult LT programme. The paediatric programme LT was preserved despite a decrease in organ offering and retrieval nationally plus limitations on adult intensive care resources at a regional level. Overall, paediatric LT outcome remained very good.

\section{GOSH-UCLH TRANSITION IN NEUROGASTROENTEROLOGY AND MOTILITY: EMBRACING READY STEADY GO HELLO} ${ }^{1}$ Maha Elhusseiny, ${ }^{1,2}$ Leanne Goh, ${ }^{2}$ Edward Gaynor, ${ }^{2}$ Anna Rybak, ${ }^{2}$ Keith Lindley,
${ }^{1,2}$ Fevronia Kiparissi, ${ }^{1}$ Terry Segal, ${ }^{1}$ Sara McCartney, ${ }^{1}$ Lee Martin, ${ }^{1}$ Joanna Begent,
${ }^{2}$ Osvaldo Borrelli, ${ }^{1}$ Natalia Zarate-Lopez. ${ }^{1}$ University College London Hospital; ${ }^{2}$ Great
Ormond Street Hospital

\subsection{6/flgastro-2021-bspghan.4}

Introduction/Background In the last decade, Neurogastroenterology \& Motility (N\&M) has become a major clinical speciality in both paediatric and adult gastroenterology, encompassing gastrointestinal (GI) conditions from classic motility disorders, such as achalasia and intestinal pseudo-obstruction, to functional GI disorders (FGID). The latter represents one of the most challenging and common groups of disorders managed by both primary care practitioners and GI specialists. Appropriate transition is particularly challenging due to the complexity of this group of patients and a holistic approach, including dietetic, psychology, psychiatry, social work, physiotherapy and occupational therapy, is characteristically required. In the last year, a formal transition pathway has been developed between GOSH and UCLH N\&M services.

Aim We aimed to review the clinical features and the complex needs of a group of young people transitioned from a paediatric to adult N\&M.

Method All patients aged 13-24 transitioned to the UCLH N\&M service over a period of 6 months were retrospectively reviewed. Demographic data, diagnosis, diet, biopsychosocial complexities, including multiple speciality involvement, polypharmacy, and known psychiatric and/or neurodevelopmental disorders were reported.

Results Ninety-two patients $(70.7 \%$ Female) under the neurogastroenterology adolescent and young-adult service were included into the analysis, of which $72.8 \%$ were $13-18$ years of age, 23.9\% 19-22 years of age and 3.3\% 22-24 years of age. Twenty-seven patients $(29.3 \%)$ were diagnosed with an underlying motility disorders, $59(64.1 \%)$ with FGID and 8 (8.7\%) with GI-allergy. The majority of patients $(80.5 \%)$ were under one or more additional medical specialities, with $28.3 \%$ under 3 or more medical specialities. Polypharmacy was common within this cohort, with $61 \%$ of patients being on 3 or more medications, whilst only $1.1 \%$ of patients required no medication to manage the symptoms. The majority of patients $(56.5 \%)$ had mental health or developmental needs, such as anxiety (25\%), depression (12\%), eating disorders (5.4\%), and learning difficulties (14.1\%). Psychological interventions were necessary in $69 \%$ of the patients, whilst dietetic interventions in $76 \%$ of patients.

Summary/Conclusion Our study confirms the need for multidisciplinary support from the specialist adolescent medicine team to provide medical and psychological care when highly demanding complex patients are transitioned between a paediatric and an adult N\&M services. Our data strongly supports a specialist adolescent transition hub model to ensure the delivery of developmentally appropriate healthcare, which has been shown to improve long-term health outcomes for young people with complex conditions. Although N\&M expertise at 